\title{
Determinación social de la resistencia para la práctica sexual protegida en parejas en situación de migración internacional, México-Estados Unidos. Propuestas para implementación de política pública*
}

Social determination for refusal to protected sexual practices in international migrant couples, MexicoUSA. Proposals for public policies implementation

\section{Determinação social da resistência às práticas sexuais protegidas em casais em situação de migração internacional, México-Estados \\ Unidos. Propostas para a implementação de políticas públicas}

Recibido: 13 de Julio de 2019. Aceptado: 28 de Enero de 2020.

Publicado: 1 de Octubre de 2020.

DOI: https://doi.org/10.11144/Javeriana.rgps19.dsrp

\author{
Yesica Yolanda Rangel Flores ${ }^{a}$ \\ Universidad Autónoma de San Luis Potosí, México \\ ORCID: https://orcid.org/0000-0001-5673-6891 \\ María Cecilia Costero Garbarino \\ El Colegio de San Luis A.C., México \\ ORCID: https://orcid.org/0000-0002-6800-3537
}

Para citar este artículo Rangel YY, Costero MC. Determinación social de la resistencia para la práctica sexual protegida en parejas en situación de migración internacional México-Estados Unidos. Propuestas para implementación de política pública. Rev Gerenc Polit Salud. 2020;19. https://doi.org/10.11144/ Javeriana.rgps 19 .dsrp

\footnotetext{
a Autora de correspondencia. E-mail: yrangelmaestria@hotmail.com
} 


\section{Resumen}

América Latina y México han evidenciado cambios en el comportamiento epidemiológico de la infección por Virus de Inmunodeficiencia Humana, especialmente en mujeres heterosexuales, rurales y unidas en relaciones estables. Este fenómeno ha sido relacionado con la migración internacional. El objetivo de esta investigación es analizar las condiciones que influyen en la resistencia para la práctica de encuentros sexuales protegidos en parejas en situación de migración internacional (México-Estados Unidos). Se trata de un estudio cualitativo con enfoque en Teoría Fundamentada en el que se entrevistó a 12 mujeres, concubinas o esposas de personas con patrón de migración circular hacia Estados Unidos. Se identificó que las condicionantes que se asocian con esta resistencia derivan de ausencias en la implementación de políticas públicas para garantizar a las mujeres una educación sexual integral, de la imposibilidad de alcanzar independencia financiera, así como de la falta de acceso a una comunicación del riesgo de contagio del VIH/sida desde una perspectiva intercultural. Dado que no existe un antecedente de educación sexual integral, las mujeres reproducen imaginarios que estigmatizan el condón y a las personas que lo usan. De igual forma, la dependencia financiera contribuye a que se vean obligadas a continuar compartiendo sexualmente con personas que asumen prácticas arriesgadas. Finalmente, esta población no recibe una consejería en salud adaptada a su contexto que le permita desarrollar una percepción realista del riesgo.

Palabras clave: Emigración e inmigración, sexualidad, prevención primaria, migrantes, determinantes sociales de la salud.

\section{Abstract}

Latin-America and Mexico have presented changes in the epidemiologic behavior of the infection by Human Immunodeficiency syndrome, especially in heterosexual women, rural workers with stable relationships. This phenomenon has been related to international migration. The objective of this research is to analyze the conditions which influence rejections to protected sexual intercourses in international migrant couples (Mexico-USA). A qualitative study with Grounded Theory orientation was applied. 12 women, concubines or wives of people with migrant patterns to the USA were interviewed. It was identified that conditions associated with this rejecting derive from the absence of implementation of public policies to guarantee a comprehensive sexual education; from the impossibility of reaching financial autonomy; and from a lack to access to the communication of the VIH contagious risk with an intercultural perspective. Since there is not a precedent of comprehensive sexual education, women reproduce cultural imaginaries that stigmatize the preservative and people who use it. Equally, financial dependence forces women to keep sexual intercourses with people who take risk practices. Finally, this population doesn't receive proper advice adapted to their context that allows them to developpe a realistic perception of the risk.

Keywords: Immigration and emigration, sexuality, primary prevention, migrants, social determination of health.

\section{Resumo}

América Latina e México têm evidenciado mudanças no comportamento epidemiológico da infecção por Vírus da Imunodeficiência Humana, especialmente em mulheres heterossexuais, rurais e unidas em relações estáveis. Este fenómeno tem sido relacionado com a migração internacional. O objetivo desta pesquisa é analisar as condições que influenciam a resistência à prática dos encontros sexuais protegidos em casais em situação de migração internacional (México-Estados Unidos). Trata-se de um estudo qualitativo com enfoque em Teoria Fundamentada no qual foram entrevistadas 12 mulheres, concubinas ou esposas de pessoas com padrão de migração circular a Estados Unidos. Identificou-se que as condições que se associam com esta resistência derivam da ausência de implementação de políticas públicas para garantir às mulheres uma educação sexual integral, da impossibilidade de atingir independência financeira, bem como da falta de acesso a uma comunicação do risco de contágio do HIV desde uma perspectiva intercultural. Dado que não existe um antecedente de educação sexual integral, as mulheres reproduzem imaginários que estigmatizam o preservativo e às pessoas que o usam. Igualmente, a dependência financeira contribui a que as mulheres tenham que continuar compartindo sexualmente com pessoas que assumem práticas arriscadas. Finalmente, esta população não recebe um aconselhamento em saúde adaptada a seu contexto que lhe permita desenvolver uma percepção realista do risco.

Palavras-chave: Emigração e imigração, sexualidade, prevenção primária, migrantes, determinantes sociais de saúde. 
Determinación social de la resistencia para la práctica sexual protegida en parejas en situación de migración internacional, México-Estados Unidos. Propuestas para implementación de política pública

\section{Introducción}

México y, en general América Latina, han evidenciado un importante cambio en el comportamiento epidemiológico de la infección por el Virus de Inmunodeficiencia Humana (VIH), la cual paulatinamente ha ido ganando presencia en mujeres heterosexuales, rurales y unidas en relaciones estables. Desde la investigación social y epidemiológica este fenómeno se ha relacionado con la migración internacional (1).

Chiapas y Guerrero, dos estados de México reconocidos por su densidad rural e indígena, son territorios en los que el VIH ha ganado terreno como causa de enfermedad y muerte entre las mujeres. Para 2016, la infección por VIH fue identificada como la tercera causa de muerte entre las mujeres indígenas, y la séptima entre las no indígenas en la región de Guerrero (2). En los Altos de Chiapas, para 2012, un 43\% de las personas totales diagnosticadas de VIH eran mujeres indígenas (3).

Las determinantes que contribuyen al vínculo infección-ruralidad son diversas y están asociadas a condiciones estructurales como el analfabetismo, la pobreza, la violencia y la construcción social de género (4). En este contexto, se requieren nuevas investigaciones para comprender las limitaciones que tienen las mujeres rurales para percibir el riesgo y protegerse ante el mismo; poco se ha investigado, por ejemplo, sobre las razones de la resistencia que estas mujeres tienen para aceptar el riesgo. Mary Douglas señala que aceptar o no la existencia de un riesgo deriva de un marco moral sustentado en los valores sociales de cada grupo social (5); en este sentido, asumir "que se está expuesta a..." redefine a los grupos y colectivos. La infección por VIH vinculada históricamente con prácticas homosexuales y liberales, constituye un riesgo que se torna difícil de aceptar desde lo moral para un grupo de mujeres cuya imagen colectiva es casi asexual o con una práctica sexual justificada en el marco de la reproducción.

Para la antropología del riesgo, el centro medular para estimar la gestión que del riesgo pueda elaborarse desde lo individual y lo colectivo consiste en comprender cuales son los procesos que favorecen o entorpecen la aceptación del riesgo; entendiendo dicha "gestión" como una serie de estrategias que las personas despliegan para reducir la probabilidad de malos resultados al estar en contacto con la amenaza (5), en este caso específico, para sugerir, solicitar o exigir el uso de condón a sus parejas, lo que llamamos en este artículo Práctica sexual protegida (PSP).

Generar conocimiento sobre cuáles son las condiciones estructurales que potencian la agencia para gestionar la PSP, -entendiendo estas condiciones estructurales como todas aquellas que contribuyen a la continuidad de la explotación o marginación de grupos de personas a favor de los grupos hegemónicos de poder-, permite aportar a la construcción de políticas que posibiliten que las mujeres den cuenta del riesgo y gestionen la PSP, entendiendo que esto generará beneficios tanto en el país de origen como en los de destino, reduciría la propagación transfronteriza del VIH y en el caso de personas infectadas, reduciría prácticas que potencian el riesgo de reinfección o co-infección con virus como el de la Hepatitis $\mathrm{C}$, fenómeno que ha ganado escenario en los últimos tiempos y que complejiza el pronóstico de la enfermedad, dado 
que deriva en una progresión más rápida hacia la muerte (6), un incremento en los costos del tratamiento (7) y una merma significativa en la calidad de vida de las personas infectadas (8).

En el contexto antes señalado, el objetivo de esta investigación fue analizar las condicionantes que influyen en la determinación social de la resistencia para la PSP en parejas rurales en situación de migración circular México-EUA, en una comunidad transnacional del centro norte de México.

\section{Antecedentes}

Los estudios que se han desarrollado sobre VIH-feminización-ruralización desde una perspectiva de género, han colocado en la palestra de qué forma la construcción social de las masculinidades -sustentadas en el machismo- desalientan la PSP. En este sentido y sin embargo, poco se ha documentado sobre las limitaciones que enfrentan las mujeres rurales para gestionar el riesgo. Lo anterior queda evidenciado cuando en la búsqueda de estudios sobre agencia para confrontar el riesgo frente al VIH, damos cuenta que en la investigación subyace un imaginario que no reconoce a las mujeres rurales como actoras capaces de gestionar el riesgo, contrario a lo que sucede con adolescentes escolarizadas o universitarias a quienes se les reconoce como actoras capaces de gestionar su riesgo, una diferenciación refuerza en el imaginario la idea equivocada y victimista de que las mujeres rurales e indígenas carecen de agencia frente a este tipo de riesgos (9).

Los pocos estudios que se han realizado con mujeres rurales o indígenas desde una perspectiva de agencia han documentado que la idea "de progreso" (como utopía) condiciona que las mujeres subestimen los riesgos que trae consigo la movilidad. Además, existe una barrera cultural para comprender que los territorios entre los que sus parejas se mueven son distintos culturalmente hablando y que esto implica la existencia de riesgos que se trasladan de un contexto a otro con el fenómeno de la movilidad migratoria (10), por ejemplo, que los varones en el contexto de la migración México - Estados Unidos, se han documentado más dispuestos a sostener encuentros homosexuales (11).

En otros estudios con población rural o indígena, se ha documentado que el machismo entendido como un imaginario cultural que considera a las mujeres seres inferiores con relación a los hombres-, motiva la resistencia de los varones a asumir la PSP, así como para acercarse a los servicios de salud (12). En estos espacios rurales el constructo social de la feminidad dificulta el desarrollo de agencia de las mujeres sobre sus derechos sexuales y reproductivos, los que con frecuencia se ven violentados aludiendo "usos y costumbres" (13).

Las limitaciones que enfrentan las mujeres para gestionar la PSP son diversas. Un estudio realizado con mujeres mexicanas documentó que antes de saberse infectadas las mujeres no habían hecho uso consistente del condón, pero incluso tras el diagnóstico confirmatorio de 
VIH, las mujeres optaban por no usar condón en el encuentro sexual porque lo asociaban con la aceptación pasiva de infidelidad por parte de sus parejas, se negaban a la aceptación del diagnóstico, o bien porque se adjudicaban o atribuían a sus parejas, propiedades morales incompatibles con el desarrollo de la enfermedad (14).

Adicionalmente, no se encuentran estudios que aborden la ausencia de agencia para gestionar el riesgo desde una mirada a las condiciones estructurales que enmarcan la vida de las mujeres rurales, así como de aproximaciones con mujeres rurales cuyas parejas participan de manera activa en la migración internacional con patrón circular (van y vuelven). Este vacío en la literatura nos hace preguntar ¿Cuáles son las condicionantes que influyen en la resistencia para la práctica de encuentros sexuales protegidos en parejas en situación de migración internacional México-Estados Unidos?

\section{Metodología}

Investigación de tipo cualitativo orientada desde la perspectiva de la determinación social de la salud (DSS), que problematiza los fenómenos epidemiológicos a partir de contextos socio históricos específicos, en los que los procesos de salud y enfermedad están enmarcados en "relaciones sociales que separan a la población en clases, cruzadas por relaciones etnoculturales y de género" (15).

Las participantes fueron 12 mujeres, esposas y concubinas de hombres migrantes insertos en patrones de migración circular: que van y viene de México y Estados Unidos, seleccionadas a través de muestreo teórico. Este tipo de muestreo consiste en incorporar inicialmente personas que vivan el fenómeno, e ir completando las entrevistas con actoras que aportan a la compresión más profunda del mismo, y de acuerdo con la información que comparten las informantes iniciales, es posible construir conocimiento teórico desde lo emergente (16). El número de informantes se limitó por saturación teórica, suspendiendo las entrevistas una vez que no emergían datos nuevos, y se consideró las categorías fortalecidas para orientar el análisis (17).

La participación se convocó mediante invitación general a través de actores clave como auxiliares de salud, procurando mujeres cuyas parejas participaran en migración hacia Estados Unidos con periodos de retorno mínimo de una vez al año y una antigüedad en esta experiencia mínima de cinco años. Aunque de manera inicial se pensó en limitar la participación a mujeres en edad reproductiva (15-45 años), finalmente la convocatoria se realizó de manera general bajo la primicia de desbiologizar la sexualidad y dar cuenta que la sexualidad no se acota en la posibilidad reproductiva.

La información se obtuvo mediante entrevista, la cual se dirigió a partir de un guión de preguntas semiestructurado, estas fueron realizadas en los domicilios de las mujeres y en sitios públicos (canchas, parque, salón ejidal), estos últimos fueron los preferidos por mujeres que cohabitaban con suegras. La duración de las entrevistas fue en promedio de 45 minutos y 10 de 12 participantes requirieron más de un encuentro para compartir la información, toda vez que 
les fue complicado hablar de sus prácticas sexuales y particularmente de la violencia asociada a esta desde un primer encuentro con las investigadoras.

Las entrevistas se audio-grabaron previo consentimiento informado por escrito, una vez transcritas se desecharon los audios para garantizar la confidencialidad de la información. Finalmente, el análisis se realizó con el enfoque de Strauss y Corbin, las dos investigadoras aplicaron la codificación abierta, axial y selectiva a los datos; primero se revisaron varias veces para reducirlos a códigos vivos y teóricos, posteriormente se conglomeraron en subcategorías y categorías sobre las cuales se dio curso al contraste y análisis teórico. La codificación axial posibilitó establecer relaciones teóricamente explicadas entre categorías y subcategorías; y finalmente, en la codificación selectiva se llegó hasta la categoría central y se rastreó sus interrelaciones.

Este estudio se enmarcó para la procuración de aspectos éticos y legales en la Declaración de Helsinki y la Ley General de Salud en México (LGSM). Fue revisado y aprobado por el Comité Estatal de los Servicios de Salud, asentado en el expediente 008603. Se documentó el consentimiento informado de las participantes libres de situación de coacción, se garantizó su derecho de renunciar a participar en el estudio en el momento que lo decidieran y se les aseguró la salvaguarda de la identidad usando nombres ficticios en la publicación de los resultados.

\section{Resultados}

Este apartado está estructurado de tal manera que el lector dé cuenta como la agencia de las mujeres frente a los riesgos no emerge como una elección individual pura sino como resultado de carencias reales y simbólicas, incluidas las ausencias en materia de política pública. En primer lugar se presenta la caracterización sociodemográfica de las informantes, posteriormente se desarrolla la categoría central, y finalmente se problematizan las ausencias o fragilidades que derivan de las políticas públicas, impulsadas en tres esferas reconocidas como determinantes en el empoderamiento de las mujeres: 1) la educación sexual integral para que la mujer decida sobre su propio cuerpo; 2) el fomento de la independencia financiera, que les posibilita decidir sobre su proyecto de vida; y 3 ) el acceso a la información sobre el riesgo específico de VIH/ sida, lo cual determina la percepción del riesgo.

Enfocar los resultados desde una perspectiva de crítica y de aporte a las políticas públicas surge de la petición de la OMS respecto a que las problemáticas en salud se reconozcan socialmente complejas y en dicho sentido inter-dependientes con otros sectores como la educación y el desarrollo social (18). 
Determinación social de la resistencia para la práctica sexual protegida en parejas en situación de migración internacional, México-Estados Unidos. Propuestas para implementación de política pública

\section{Caracterización sociodemográfica de las participantes}

El rango de edad fue de 23 a 53 años, y el promedio de 37 . En cuanto a la escolaridad, ocho contaban con la educación primaria, tres con secundaria y una con primaria incompleta. Sobre la ocupación, la totalidad enunció las labores de casa como actividad principal y las 12 eran beneficiarias del Seguro Popular. El rango de tiempo en pareja con la persona en situación de migración iba de ocho a 21 años, el promedio fue de 17.

En la codificación abierta surgieron 412 códigos vivos, los cuales se combinaron en 12 subcategorías, que se conglomeraron para la codificación selectiva en tres categorías para análisis.

\section{Categoría central: resistencias para la práctica sexual protegida (PSP)}

Se documentaron múltiples narrativas que evidencian la resistencia para la PSP con parejas estables. Resultó relevante el hecho de que pese a estar conscientes de los riesgos que implica la migración para la transmisión de Infecciones de Transmisión Sexual (ITS), las mujeres y sus parejas se resisten a usar condón durante sus encuentros sexuales.

Esta consciencia de riesgo se evidenció en narrativas como la siguiente:

Allá existe más esa enfermedad que aquí, mi esposo me platica que los meten en una gallinera antes de terminar de pasarlos y que les llevan a personas a darles esos servicios [prostitución] por lo mismo que saben que 'eso' para un hombre es necesidad... lo malo es que nomás no se arriesgan ellos, lo arriesgan a uno, hasta a los hijos, a toda la familia (P3).

Las mujeres se colocan en una posición distinta a la que colocan a sus parejas con relación a la amenaza, esto hace necesario aclarar la diferencia epistémica entre dos conceptos que suelen utilizarse de manera indistinta: riesgo y vulnerabilidad. Ante el riesgo los sujetos poseen autonomía para tomar decisiones y confrontar la amenaza; en cambio, la vulnerabilidad aborda situaciones en las que, frente a lo amenazante, se carecen de agencia para actuar respecto a la misma (19). Desde esta premisa, las mujeres no logran situarse en una posición de riesgo sino más bien de vulnerabilidad, puesto que no se reconocen con la autonomía para gestionar la PSP. Sin embargo, estas mujeres podrían desarrollar autonomía, si bien no en materia de contener la posibilidad de que sus parejas tengan encuentros sexuales con otras personas, sí para asegurarse que estos usen condón al estar sexualmente con ellas; lo interesante es que esta protección no solo no es contemplada como una posibilidad, sino que resulta indeseable para ellas mismas.

La PSP es indeseable porque la contemplan como una muestra de que "autorizan" a sus parejas el encuentro sexual con terceras personas, como se ve en la siguiente narrativa.

Nunca le he dicho a él que use condón. Lo usó un tiempo, pero fue porque él dijo que me hacía daño tomar tantas hormonas... sabe qué haría si me encontrará condones en la casa, yo creo que me diría ¿Y tú porque 
tienes? ¿Con quién los usastes? Si creo que se enojaría; yo también me enojaría si le encontrara condones porque ¿pa' que los quiere? Porque entonces ha tenido relaciones con otra, si me molestaría porqué sería como una prueba de que si anda de demás ¿no? (P5).

A continuación, se profundiza en las condicionantes que influyen en la resistencia para la PSP, las cuales visibilizan ausencia de políticas dirigidas a garantizar: 1) Educación sexual integral, 2) Independencia financiera en las mujeres, y 3) Acceso a una comunicación del riesgo desde una perspectiva intercultural.

\section{Educación sexual integral}

En México pese a que a partir de la última década se ha trabajado en la incorporación de un modelo de educación sexual integral desde la educación básica (20), la realidad es que desde las narrativas que las informantes construyen, prevalece la percepción de una sexualidad generizada, enmarcada en el imaginario de la reproducción y sostenida en aspectos morales más que científicos. La sexualidad femenina se vislumbra como una más de las responsabilidades que adquieren con el matrimonio, con una finalidad reproductiva y dependiente de las condiciones que sus parejas establezcan. Por su parte, la sexualidad masculina se representa como una práctica instintiva, que poco puede regularse, incluso por los mismos hombres. Ejemplo de lo anterior se puede apreciar en las siguientes narrativas.

Con referente a su propia sexualidad:

Yo estoy ahora sí que como él diga, en veces llega y no sé si me va a usar, pero una tiene que estar, aunque no esté de guenas $(\mathrm{sic}$ )... Ahora sí que una lo hace para que ellos no busquen afuera lo que deberían tener en la casa, luego con ese pretexto es que andan demás.

Con referencia a la sexualidad de sus parejas: "Yo miro que para ellos eso es una necesidad, no pueden estar sin eso, ellos no lo pueden controlar, es que se ponen de malas si ¡no!”.

La representación condón-promiscuidad/infidelidad se instituye en un obstáculo cultural, moral y social para la gestión de la PSP, reta representaciones del orden social en dos sentidos, por un lado, cuestiona la vigencia de una de las instituciones más valoradas en nuestro contexto, la monogamia y la exclusividad sexual que se asocia a esta. Por otro, el hecho de que las mujeres muestren conocimiento sobre sexualidad y métodos de protección, les obliga a renunciar a ser contempladas como "inexpertas" o "decentes", dos valores simbólicos que representan para muchas la única propiedad que poseen para resultar "dignas de ser esposas".

Aceptar o solicitar que sus parejas usen condón las obliga a recolocarse en la escala de estatus moral, toda vez que el estigma no se posiciona solo en el condón sino en la mujer que gestiona 
Determinación social de la resistencia para la práctica sexual protegida en parejas en situación de migración internacional, México-Estados Unidos. Propuestas para implementación de política pública

la PSP, toda vez que su uso se significa como una práctica inmoral puesto que se asocia con prácticas sexuales casuales, extramaritales o con sujetos clasificados "de riesgo".

Para ejemplificar el estigma que el condón llega a tener en los contextos de estas mujeres, basta recuperar el rechazo que ellas muestran a la posibilidad de que sus parejas porten condones, una acción que lejos de representarles tranquilidad les significa una agresión que contraviene su imaginario de monogamia.

Si él me dijera que quiere usar condón y lo trajiera sí me enojaría, porque pensaría que eso también lo usa estando allá, sí lo tomaría a mal porque eso querría decir que él se está metiendo con otra y yo no sé, a ver dígame ¿a mí de que me sirve que se meta con otra y use condón si de todas formas se está metiendo con otra persona? yo de todas maneras me enojaría (P7).

\section{Autonomía e independencia financiera}

México es uno de los países con mayor desigualdad de género en el acceso al empleo, se sitúa en el lugar 128 en la lista de 144 países en función de la brecha salarial entre los géneros (21). Lo anterior explica que uno de los principales temores de las mujeres sea el miedo a no cumplir con la expectativa sexual de sus compañeros, principalmente cuando en el marco de la migración, se saben expuestas a ser abandonadas dentro de un contexto con escasas oportunidades de empleo.

Cuando él viene yo trato de tenerlo bien, su comida, su cuidado, lo que él necesita (risas) ¡Todo! (haciendo referencia a lo sexual), porque hay hombres que por lo mismo luego no regresan, porque hallan una mujer allá y hacen otras familias, así le pasó a la señora, por lo mismo que aquí ya no se encontraba, o sea, ya cuando estaba con ella no le gustaba (no le complacía sexualmente), ya no regresó.

La infidelidad figura como una situación que es común en sus vidas, sabiendo de casos en los que los encuentros extramaritales ocurren en las localidades de destino y los trayectos migratorios, aunque también, su falta de deseo de "confirmar", las hace vivir en la incertidumbre sobre las prácticas sexuales que sus parejas, este "desconocimiento" influye en el hecho de que no consideren necesario la PSP, a su retorno.

Él no me ha dicho nunca si (fue infiel), pero yo pienso que sí por lo mismo que él hacía, en veces le llamaba y me contestaban los amigotes y me decían 'no es que ya no te manda dinero porque ya tiene otra acá y embarazada, yo nunca lo confirmé, pero eso dijieron (sic) (P5).

Otras mujeres relataron conocer la práctica de encuentros extramaritales por sus parejas en las localidades de destino, sin embargo, parecían más preocupadas por no ser abandonadas (o desplazadas por la nueva mujer) que por la probabilidad de ser contagiada.

\section{Acceso a la comunicación del riesgo}

La mayoría refirió no haber sido comunicada sobre el riesgo potencial de ser contagiada de una ITS, por parte de personal de salud u otros funcionarios, y aun cuando la mayor parte de 
ellas conoce aspectos asociados a la enfermedad; en general, no dimensionan el riesgo al que están expuestas desde su posición. Lo anterior ha sido señalado como consecuencia de que esta enfermedad en particular se haya configurado desde la política pública como una enfermedad asociada a grupos y prácticas sexuales estigmatizadas, con prevalencia en el ámbito urbano y las sociedades desarrolladas, invisibilizando de esta manera el riesgo que implica en comunidades rurales, pobres e indígenas (22).

Algunas refirieron haber sido objeto de pruebas de detección rápida sin comprender a fondo la condición que las hacía objeto de tales procedimientos, otras llegaban a referirse violentadas y en desacuerdo con estas pruebas, así como con los discursos desde los cuales se pretende despertar una consciencia sobre el riesgo, esto acontece particularmente por el hecho de que se trata de una enfermedad altamente estigmatizada desde lo moral (23).

Esta ausencia de agencia y de consciencia de riesgo se hace evidente cuando las mujeres no solo rechazan la posibilidad de participar en pruebas de detección oportuna sino que manifiestan incomodidad con el discurso sanitario, respecto a un riesgo que consideran ajeno a sus experiencias.

A veces si me cansa que me digan que yo tengo más riesgo porque él anda en el otro lado, yo entiendo que es el trabajo de ellos (personal de salud) inculcarle a uno, porque a veces en ciertas cosas sí somos bien cerradas, pero deberían pensar que no, nomás las que tenemos esposos allá tenemos ese riesgo (P7).

El relato evidencia cómo el discurso sanitario entra en conflicto con las representaciones que estas mujeres poseen respecto a la enfermedad y su sexualidad, en este sentido, aun cuando muchas de ellas acepten escuchar el mensaje del personal de salud respecto al riesgo y realizarse las pruebas de detección oportuna, dicha "aceptación" acontece dentro de un marco más interesado por evadir el conflicto con autoridades institucionales que como resultado de una verdadera conciencia respecto al riesgo.

Las narrativas compartidas permiten dar cuenta cómo la salud y su procuración, no se acotan al dominio de la individualidad y la voluntad, sino que están insertas en contextos determinados socialmente en los que priman relaciones de poder desiguales (24). Los resultados exigen también problematizar las construcciones culturales que prevalecen en cada población y considerar la existencia de desigualdades estructurales que colocan a las personas en distintas posiciones respecto a los riesgos. En este sentido, resulta pertinente implementar políticas públicas fundamentadas en un enfoque interseccional, en el que la raza, las diferencias educacionales y la clase social posibilite intervenir desde un paradigma que dé cuenta que la inequidades en salud están basados en las condiciones de vida (25).

La migración, como otros fenómenos sociales contemporáneos, genera impactos globales que deben visibilizarse y preverse (o en su defecto atenderse) a través de políticas públicas cuyas 
Determinación social de la resistencia para la práctica sexual protegida en parejas en situación de migración internacional, México-Estados Unidos. Propuestas para implementación de política pública

acciones impacten no sólo en quienes participan de manera activa en los procesos migratorios, sino también y quizá principalmente en las personas que sin movilizarse, enfrentan nuevos riesgos, de los que con frecuencia no son conscientes (26).

\section{Conclusiones y recomendaciones}

El objetivo de esta investigación fue analizar las principales condicionantes que influyen en la determinación social de la resistencia para la práctica de encuentros sexuales protegidos en parejas rurales en situación de migración internacional, en una comunidad transnacional del centro norte de México.

Se identificó que existe una actitud de resistencia frente a la práctica sexual protegida y que está asociada principalmente a tres situaciones:

1. No existe un antecedente de educación sexual integral, es por esto que las mujeres reproducen imaginarios que estigmatizan y enmarcan dentro de lo inmoral el uso del condón y las personas que hacen uso del mismo.

2. No hay condiciones que posibiliten la autonomía e independencia financiera en estas mujeres, en función de esto es que aun conociendo las prácticas de riesgo que sus parejas realizan y de las que pueden resultar eventualmente afectadas, "deciden" continuar compartiendo sexualmente, pues consideran que no existen los medios económicos ni sociales para desempeñarse como sujetas autónomas en sociedad.

3. No tienen acceso a una consejería en salud que les posibilite construir una percepción objetiva del riesgo.

Con este marco, y a partir de los hallazgos reportados se identifican pertinentes las siguientes recomendaciones generales:

Para generar indicadores de seguimiento del fenómeno es preciso incluir en la Encuesta Nacional sobre la Dinámica de las Relaciones en los Hogares (ENDIREH) una o más variables que posibiliten identificar a las parejas que están involucradas en procesos de migración. Aunque actualmente INEGI realiza la Encuesta Nacional de la Dinámica Demográfica en la cual explora la migración interna y externa, estudios como este documentan la importancia de explorar el antecedente de migración vinculado a la vulnerabilidad que las mujeres enfrentan respecto a violencias específicas como la sexual, entendiendo esta como:

cualquier acto que degrada o daña el cuerpo y/o la sexualidad y que por tanto atenta contra su libertad, dignidad e integridad física.... expresión de abuso de poder que implica la supremacía masculina sobre la mujer, al denigrarla y concebirla como objeto (27 s.p.).

Construir un marco de coordinación intersectorial para combatir las violencias contra las mujeres en situación de migración, para visibilizar las vulnerabilidades que viven las que esperan el retorno de sus parejas en las comunidades de origen, toda vez que los que existen 
actualmente se centran en las personas que migran y no en quienes esperan su retorno en las comunidades transnacionales.

A continuación se exponen las recomendaciones específicas derivadas de la presente investigación.

Recomendaciones para fomentar el desarrollo de agencia sexual y reproductiva en las mujeres:

Implementar con rigurosidad tamizajes de identificación de violencia en este grupo en particular, las mujeres rurales e indígenas son susceptibles a normalizar la violencia o no identificar cuando esta ocurre en el plano sexual por parte de sus esposos o concubinos.

Es conveniente continuar trabajando en la universalización de las pruebas rápidas y la promoción del uso de preservativo, es pertinente focalizar los esfuerzos en monitorear la educación sexual y consejería que en materia de salud sexual y reproductiva reciben las mujeres rurales e indígenas.

Monitorear de manera permanente los programas de educación sexual que se imparten dentro de las instituciones del Estado y capacitar a los maestros rurales para abordar estas temáticas desde una perspectiva de derechos humanos.

Promover el acceso a la información en materia de violencia contra las mujeres, fortalecer y hacer más accesibles los mecanismos de denunciar y acceso a la justicia. Desde estas acciones las mujeres identificarán, denunciarán y saldrán de contextos de violencia que pueden enfermarles.

Priorizar la implementación de protocolos de diagnóstico de ITS en mujeres con antecedente de violencia sexual y parejas de varones en situación de migración.

Impulsar la respuesta ciudadana favoreciendo la conformación de redes de mujeres en situación de migración o parejas de hombres en migración para el empoderamiento de su agencia en la problemática.

Garantizar el derecho a la educación sexual integral y diseñar una estrategia intersectorial que posibilite que las instituciones del Estado procuren una intervención respetuosa de la autonomía de las mujeres sobre sus procesos sexuales y reproductivos.

Recomendaciones para promover la independencia emocional y financiera:

Generar mecanismos de vigilancia ciudadana hacia las políticas públicas dirigidas a garantizar derechos humanos de las mujeres rurales e indígenas. 
Impulsar acciones afirmativas que posibiliten la incorporación y permanencia de niñas, adolescentes y mujeres en el sistema educativo; y más adelante para garantizar procesos formativos y de oportunidades empresariales en hombres y mujeres, con medidas afirmativas hacia estas últimas.

Consolidar mecanismos para que las mujeres rurales e indígenas puedan conciliar la vida laboral y familiar, así como implementar acciones que apoyen el maternaje.

Recomendaciones para favorecer la percepción del riesgo y, consecuentemente, el desarrollo de agencia frente a la amenaza:

Impulsar la investigación social sobre ITS en población en situación de migración, garantizando la implementación de perspectivas que les visibilicen en situación de vulnerabilidad (género, interculturalidad y derechos humanos).

Promover campañas que aporten a la construcción social del riesgo de la población involucrada directa (quienes migran) o indirectamente (quienes les esperan) en las dinámicas de migración.

Garantizar presupuestos que prioricen el abastecimiento de pruebas rápidas y preservativos en localidades con alta incidencia de movilidad migratoria, así como en periodos de tiempo en los que se prevea dinámicas de retorno y salida.

De-construir los discursos que se han construido para comunicar el riesgo y que centran el riesgo en hombres que tienen sexo con hombres (HSH), población trans, personas dedicadas al trabajo sexual, personas usuarias de drogas inyectables, personas privadas de la libertad, víctimas de violencia sexual, personas en situación de calle, mujeres embarazadas y parejas sero-discordantes; posicionar a las mujeres en términos de vulnerabilidad y no sólo durante la atención perinatal.

Incluir las perspectivas de derechos humanos, género e interculturalidad en los programas de formación de recursos humanos en salud, con el fin de dotarles de competencias para transmitir información desde un marco que les posibilite una real incidencia y transforme la prescripción en consejería.

Generar foros que promuevan diálogos interculturales en temas de salud, particularmente en temas que -como este- se sustentan en una serie de prejuicios morales.

El presente estudio resulta relevante porque contrario a lo que se ha hecho en la mayor parte de la literatura, la situación de vulnerabilidad que enfrentan estas mujeres no se acota al cuerpo en su dimensión biológica sino que tiene que ver con cómo lo político se encarna en las posibilidades que sus cuerpos generizados tienen dentro de una sociedad en la que los imaginarios patriarcales merman las oportunidad de desarrollo, salud y vida; no siempre de manera tácita sino también 
simbólica a través de la generación e implementación de políticas que restan autonomía y agencia incluso sobre sus propios cuerpos.

Los resultados recuperados evidencian la necesidad de continuar haciendo investigación sobre el comportamiento epidemiológico del VIH/sida desde perspectivas humano sociales, que coloquen el foco en el tema del respeto a los derechos humanos, la perspectiva intercultural y el reconocimiento de que las construcciones sociales de género generan desigualdad e inequidad en las agencias para confrontar las amenazas.

\section{Referencias}

1. Viani R, Araneta M, Spector S. In-country migration and risk factors for HIV acquisition among pregnant women in Tijuana, Mexico. J Int Assoc Provid AIDS Care. 2016;15(3): 228-231. https://doi.org/10 $.1177 / 2325957414539043$

2. Freyermuth G. El derecho a la protección a la salud en mujeres indígenas. Análisis nacional de casos desde una perspectiva de derechos humanos. México: Centro de Investigación y Estudios en Antropología Social (CIESAS) y Comisión Nacional de Derechos Humanos (CNDH); 2016.

3. Muñoz R. Atención médica, adherencia terapéutica al tratamiento antirretroviral y discriminación. Algunas problemáticas en la atención a personas que viven con VIH-sida, desde una perspectiva antropológica. Revista Pueblos y Fronteras Digital. 2014;9(17): 95-115. http://dx.doi.org/10.22201/ cimsur.18704115e.2014.17.65.

4. Juan B, Rangel Y, Castillo L, Cacique L. Ser mujer indígena, vivir con VIH y violencia de pareja: una triple vulneración frente al derecho a la salud. Index Enfermería. 2018;27(3): 161-165. http://scielo. isciii.es/scielo.php?script=sci_abstract\&pid=S1132-12962018000200012

5. Douglas M. La aceptabilidad del riesgo según las ciencias sociales. Barcelona: Paidós Editorial; 1996.

6. Croxford S, Kitching A, Desai S, Kall M, Edelstein M, Skingsley A. Mortality and causes of death in people diagnosed with HIV in the era of highly active antiretroviral therapy compared with the general population: an analysis of a national observational cohort. Lancet Public Health. 2017;21(1): 35-46. https://doi.org/10.1016/S2468-2667(16)30020-2

7. Wolpert E, Muñoz O, Kershenobish D. Acciones prioritarias para un programa nacional de detección, tratamiento y seguimiento de pacientes con hepatitis C. Salud Pública de México. 2019;61(2): 212-216. https://doi.org/10.21149/10166

8. Haile D, Ayen W, Tiwari P. Prevalence and assessment of factors contributing to adverse drug reactions in wards of a tertiary care hospital, India. Ethiop J Health Sci. 2013;23(1): 39-48. https://www.ncb i.nlm.nih.gov/pubmed/23559837

9. Amuchástegui A. Gobernanza neoliberal en la epidemia del VIH/SIDA en mujeres en México: los efectos del paradigma de la vulnerabilidad. Estudios sociológicos. 2017;35(104): 343-371. http://dx.doi.org /10.24201/es.2017v35n104.1511 
Determinación social de la resistencia para la práctica sexual protegida en parejas en situación de migración internacional, México-Estados Unidos. Propuestas para implementación de política pública

10. Torres T, Iñiguez R, Pando M, Salazar Y. Riesgo de contagio de Infecciones de Transmisión Sexual (ITS) y VIH/SIDA desde el punto de vista de adolescentes migrantes del Estado de Jalisco, México. Revista Latinoamericana de Ciencias Sociales, Niñez y Juventud. 2009;(1): 137-156. http://www.sc ielo.org.co/scielo.php?pid=S1692-715X2009000100006\&script $=$ sci_abstract\&tlng=es

11. Núñez G. Seeve/frescos: sexualidad, género y etnicidad en los significados de las relaciones sexuales entre varones en comunidades yoeme (yaquis) de Sonora, México. Revista de Estudios de Antropología Sexual. 2013;1(4): 96-120. https://mexicana.cultura.gob.mx/en/repositorio/detalle?id=_suri:MEDIATECAARTICULO :TransObject:5bca29f87a8a0222ef132259\&word=Etnicidad\&r $=6 \& \mathrm{t}=9$

12. Gutmann M. Por mis pistolas: Sexualidad, anticoncepción y sida en México. México DF: Siglo XXI Editores; 2016.

13. Nava D, Onofre F, Báez H. Autoestima, violencia de pareja y conducta sexual en mujeres indígenas. Enfermería Universitaria 2017;14(3): 162-169. http://dx.doi.org/10.1016/j.reu.2017.05.002

14. Kendall T, Castillo A, Herrera C, Campero L. El uso inconsistente del condón en mujeres mexicanas que viven con VIH: un reto para los servicios de salud. Salud Pública de México. 2015;(57): 183-189. http://www.scielo.org.mx/scielo.php?script=sci_arttext\&pid=S0036-36342015000800012

15. Breilh J. Epidemiología del siglo XXI y ciberespacio: repensar la teoría del poder y la determinación social de la salud. Rev Bras Epidemiol. 2015;18(4): 972-982. https://doi.org/10.1590/1980-549720 1500040025

16. Glasser B, Strauss A. The discovery of grounded theory: Strategies for qualitative research. $7^{\text {th }}$ ed. New Jersey: New Jersey; 2012.

17. Strauss A, Corbin J. Bases de la investigación cualitativa, técnicas y procedimientos para desarrollar la teoría fundamentada. Bogotá: Editorial Universidad de Antioquia; 2002.

18. Organización Panamericana de la Salud, Organización Mundial de la Salud. Impulsar el enfoque de la salud en todas las políticas en las Américas: ¿Cuál es la función del sector salud? Washington D.C.: PAHO; 2015.

19. Lupton D. Risk as moral danger: the social and political functions of risk discourse in public health. Intern J Health Serv. 1998;23(3): 425-435. https://doi.org/10.2190/16AY-E2GC-DFLD-51X2

20. Salinas-Quiroz F, Rosales-Mendoza A. La agenda pendiente de la educación inicial y preescolar en México: sexualidad integral. Educación. 2016;25(49): 143-160. http://dx.doi.org/10.18800/educaci on

21. The Global Gender Gap Report 2016. World Economic Forum. Disponible en: http://www3.weforum. org/docs/GGGR16/WEF_Global_Gender_Gap_Report_2016.pdf

22. Ponce P, Muñoz R, Stival M. Pueblos indígenas, VIH y políticas públicas en Latinoamérica: una exploración en el panorama actual de la prevalencia epidemiológica, la prevención, la atención y el seguimiento oportuno. Salud Colect. 2017;13(3): 537-554. https://doi.org/10.18294/sc.2017.1120

23. Isaac M, Jorge E, Mendoza M. Significado y percepción frente al VIH/SIDA en mujeres rurales parejas de migrantes, Jalisco, México. Rev Costarric Salud Pública. 2015;24(2): 115-125. https://www.scielo .sa.cr/scielo.php?script=sci_abstract\&pid=S1409-14292015000200115\&lng=es\&nrm=iso\&tlng=es 
24. Parrini R. Biopolíticas del abandono. Migración y dispositivos médicos en la frontera sur de México. Nómadas. 2015;42: 111-127. http://www.scielo.org.co/pdf/noma/n42/n42a07.pdf

25. León P, García AJ, Pría M., Perdomo I. El abordaje de las desigualdades desde la perspectiva de las investigaciones en salud. Horizonte Sanitario. 2019;18(2): 101-109. http://dx.doi.org/10.19136/hs.a $18 \mathrm{n} 2.2432$.

26. Handley M, Sudhinarasey M. The important role of binational studies for migration and health research: A review of US-Mexico binational studies and design considerations for addressing critical issues in migrant health. Int Migr Rev. 2017;55(5): 75-121. https://doi.org/10.1111/imig. 12306

27. Ley General de Acceso de las Mujeres a una Vida Libre de Violencia. Diario Oficial de la Federación el 1 de febrero de 2007, última reforma publicada DOF 13-04-2018. http://www.diputados.gob.mx /LeyesBiblio/pdf/LGAMVLV_130418.pdf

Notas

* Artículo de investigación. 